\title{
Comparative study of protein-protein interaction observed in PolyGalacturonase-Inhibiting Proteins from Phaseolus vulgaris and Glycine max and PolyGalacturonase from Fusarium moniliforme
} Aditi Maulik, Hiren Ghosh and Soumalee Basu*

Address: Department of Bioinformatics, School of Biotechnology, West Bengal University of Technology BF-142, Salt Lake, Kolkata 700064, India E-mail: Aditi Maulik - aditi_maulik05@yahoo.co.in; Hiren Ghosh - hirenbioinfo2006@gmail.com; Soumalee Basu* - soumalee@gmail.com ${ }^{*}$ Corresponding author

from Asia Pacific Bioinformatics Network (APBioNet) Eighth International Conference on Bioinformatics (InCoB2009)

Singapore 7-II September 2009

Published: 3 December 2009

BMC Genomics 2009, I0(Suppl 3):S19 doi: 10.1186/1471-2164-10-S3-S19

This article is available from: http://www.biomedcentral.com/I47I-2/64/I0/S3/SI9

(C) 2009 Maulik et al; licensee BioMed Central Ltd.

This is an open access article distributed under the terms of the Creative Commons Attribution License (http://creativecommons.org/licenses/by/2.0), which permits unrestricted use, distribution, and reproduction in any medium, provided the original work is properly cited.

\begin{abstract}
Background: The PolyGalacturonase-Inhibiting Proteins (PGIP) of plant cell wall limit the invasion of phytopathogenic organisms by interacting with the enzyme PolyGalacturonase (PG) they secrete to degrade pectin present in the cell walls. PGIPs from different or same plant differ in their inhibitory activity towards the same PG. PGIP2 from Phaseolus vulgaris (Pv) inhibits the PG from Fusarium moniliforme (Fm) although PGIPI, another member of the multigene family from the same plant sharing $99 \%$ sequence similarity, cannot. Interestingly, PGIP3 from Glycine $\max (\mathrm{Gm})$ which is a homologue of PGIP2 is capable of inhibiting the same PG although the extent of similarity is lower and is $88 \%$. It therefore appears that subtle changes in the sequence of plant PGIPs give rise to different specificity for inhibiting pathogenic PGs and there exists no direct dependence of function on the extent of sequence similarity.

Results: Structural information for any PGIP-PG complex being absent, we resorted to molecular modelling to gain insight into the mechanism of recognition and discrimination of PGs by PGIPs. We have built homology models of PvPGIPI and GmPGIP3 using the crystal structure of PvPGIP2 (IOGQ) as template. These PGIPs were then docked individually to FmPG to elucidate the characteristics of their interactions. The mode of binding for PvPGIPI to FmPG considerably differs from the mode observed for PvPGIP2-FmPG complex, regardless of the high sequence similarity the two PGIPs share. Both PvPGIP2 and GmPGIP3 despite being relatively less similar, interact with residues of FmPG that are known from mutational studies to constitute the active site of the enzyme. PvPGIPI tends to interact with residues not located at the active site of FmPG. Looking into the electrostatic potential surface for individual PGIPs, it was evident that a portion of the interacting surface for PvPGIPI differs from the corresponding region of PvPGIP2 or GmPGIP3.

Conclusion: van der Waals and eletrostatic interactions play an active role in PGIPs for proper recognition and discrimination of PGs. Docking studies reveal that PvPGIP2 and GmPGIP3 interact with the residues constituting the active site of FmPG with implications that the proteins bind/block FmPG at its active site and thereby inhibit the enzyme.
\end{abstract}




\section{Background}

Plants are under constant threat of infections caused by pathogens that range from viruses, bacteria, fungi to nematodes and insects. The efficacy of plant defense depends on its ability to recognize a pathogen and mount the appropriate defense response. Plants often employ cell surface and intracellular receptors to detect a pathogen associated molecular pattern (PAMP) and trigger immune response against the invader. PolyGalacturonase-Inhibiting Protein (PGIP) is one among the pathogenesis-related (PR) proteins that is found at the cell surface of plant cells. It binds and inhibits the enzyme PolyGalacturonase (PG) from the invading pathogen which could be fungus, insect or bacterium, thus preventing its colonization in the host cell and hence the progress of the disease [1,2]. PolyGalacturonases (PG) are a class of pectinolytic enzyme secreted by the pathogen at the early stages of infection to depolymerize homogalacturonan (HGA), the main component of pectin in the plant cell wall [3]. HGA is the 1, 4 linked alpha-D-galactosyluronic acid polymer found in the plant cell which forms the first line of barrier and thereby plays a critical role in controlling pathogen invasion [4-7].

The interaction of the plant protein PGIP with the fungal or insect PG limits the destructive potential of the PG and leads to the accumulation of elicitor active oligogalacturonides as shown by in vitro studies [8]. Hence PGIP seems to exert a dual role during fungal attack. It limits pathogen penetration and tissue colonization by inhibiting PG activity, this in turn, favours the accumulation of oligogalacturonides, which activate a prompt defense response [9].

PGIPs belong to the Leucine-rich Repeat (LRR) super family of proteins [10] containing tandem repeats of a 20-30 amino acid stretch of the extracytoplasmic type with a consensus that bear a conserved part [LxxLxLxxNxL or LxxLxLxxCxxL ( $\mathrm{L}=\mathrm{I}, \mathrm{L}, \mathrm{V}, \mathrm{F} ; \mathrm{N}=\mathrm{N}, \mathrm{T}$, $\mathrm{S}, \mathrm{C} ; \mathrm{C}=\mathrm{C}, \mathrm{S} ; \mathrm{x}=$ any amino acid)] and a variable part $[11,12]$. The LRR-fold (Figure 1) which is believed to be specialized in protein-protein interaction is quite extensively used for the immune functions and for the recognition of non-self molecules by plants. The crystal structure of the only PGIP (PGIP2 from Phaseolus vulgaris) as well as the first LRR protein belonging to the plant-specific subfamily [13] ([ILVF] xx[ILVF] xx[ILVF] $\mathrm{x}[\mathrm{ILVF}] \mathrm{xx}[\mathrm{NTSC}] \mathrm{x}[\mathrm{ILVF}]$ [TS]GxIPxx[ILVF]Gx) reveals a typical curved and elongated shape. Eight $\beta$ strands (with one long $\beta$ strand, B1, at the N-terminal end) comprise the inner concave face of the curved surface. On the opposite side of the $\beta$ sheet, there are nine $3_{10}$ helices that are almost parallel to the $\beta$ sheet. The concave surface is known to bear residues necessary for binding and recognition specificity in this class of protein [14].

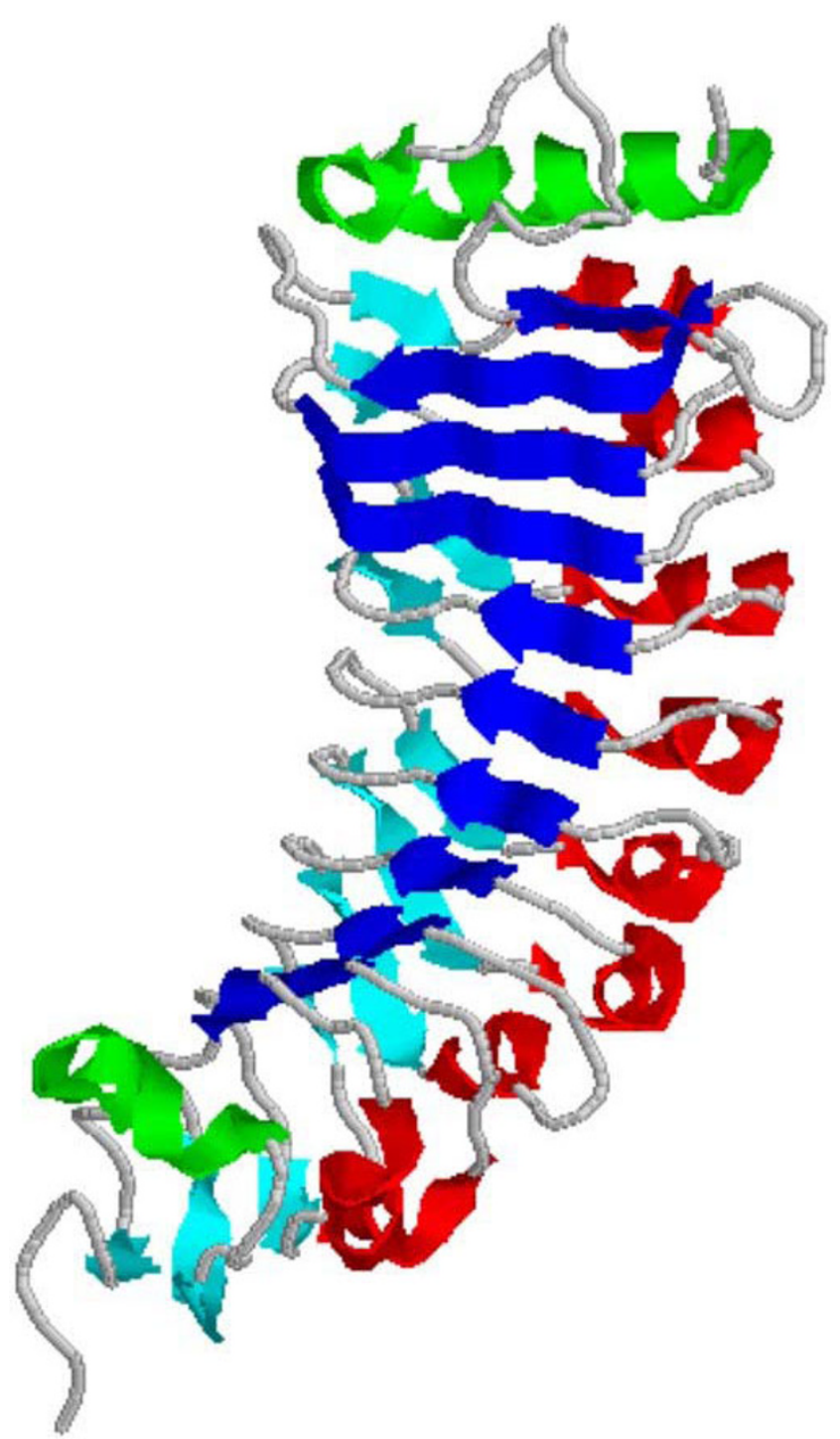

Figure I

Ribbon representation of Crystal structure of PvPGIP2 (IOGQ). The protein is composed of tandemly repeating units called LRRs. The $\mathrm{N}$-terminal and $\mathrm{C}$-terminal cysteine-rich domains are shown in green. Three structural elements characterize this fold. The $\beta$-sheet $\mathrm{BI}$ occupies the concave face of the scaffold (blue), a regular array of $3_{10}$ helices characterizes the convex face (red), and a second non-regular $\beta$-sheet (B2; cyan) is found between the two faces.

PGIPs function as very specific inhibitors [15]. PGIP from a particular plant that is able to inhibit the PG from a certain pathogen might not be able to inhibit PGs from other pathogens. Besides, for cases when a PGIP is able to inhibit PGs from different pathogens, the percent inhibition differs. Thus in plants, small gene families encode PGIP isoforms that differ in affinity and 
specificity for PGs secreted by different pathogens [1] Recognition specificity results from a few variations in the amino acid sequence of the PGIPs [16]. Plants successfully defend themselves from the attack of a wide range of PGs from pathogenic microorganisms through the role played by protein-protein interaction involving these PGIP proteins.

Significant contributions have been made with experimental studies on the different isoforms of PGIP from a variety of plants. It has been seen that the overexpression of two PGIP genes, Atpgip1 and Atpgip2 genes from Arabidopsis thaliana limits the colonization by B. cinerea and also reduces the disease symptoms [17]. In transgenic tomato and grapevine plants, a significant increase of PG-inhibitory activity and a decrease in susceptibility to $B$. cinerea have been observed. Plant resistance have been successfully enhanced by the overexpression of pear PGIP in tomato [18] and Vitis vinifera [19], and also bean PGIP in tobacco [20]. Accumulation of information on several of the properties exhibited by PGIPs have generated an interest in exploiting them as tools for enhancing plant resistance. In this regard, PGIPs of bean plant (Phaseolus vulgaris) needs special mention.

Bean PGIPs are encoded by a family of genes [21], the products of which are PvPGIP1, PvPGIP2, PvPGIP3 and $P v$ PGIP4. All of these show distinct regulation and specificity exhibiting different inhibitory capabilities against the PGs of Botrytis cinerea, Colletotrichum gloeosporioides, Stenocarpella maydis, Fusarium moniliforme and Aspergillus niger [21]. The four mature products of the pgip genes have a very high extent of sequence similarity differing between 8 to 81 amino acids. Phylogenetic analysis indicates that Pvpgip1-Pvpgip2 groups with Gmpgip3 of soybean (Glycine max), a species of the Phaseoleae tribe close to bean, which suggests that these two genes are probably closer to the ancestral gene than are Pvpgip3 and Pvpgip4 [7]. Interestingly, although PGIP1 and PGIP2 from Phaseolus vulgaris share 99\% sequence similarity, PvPGIP2 has been reported to inhibit PG from Fusarium moniliforme (Fm) while PvPGIP1 does not [16]. Strikingly, GmPGIP3 of soybean (Glycine max), shares a much lesser similarity of $88 \%$ with PvPGIP2 and yet has the ability to inhibit FmPG with an inhibition kinetics similar to that of PvPGIP2 [22]. It is thus evident that the ability of PGIP molecules to defend plants against infection is not buried in the extent of similarity shared between the sequences alone. Probable implications could be the presence of key structural features important for the necessary proteinprotein interaction that need to be conserved to preserve the function.
It would be interesting to study the molecular interaction of different PGIPs sharing different range of sequence variation, with the same PG molecule to elucidate the relation between the extent of sequence similarity and the corresponding ability/inability to inhibit PG. Three PGIPs, PvPGIP1 and PvPGIP2 from Phaseolus vulgaris and GmPGIP3 from Glycine max have been chosen for this purpose keeping in mind that they share a high level of sequence similarity and yet show distinct difference in their function as expressed through their ability/inability to inhibit the same PG from Fusarium moniliforme (FmPG). In this study, homology models of two PGIP molecules namely PvPGIP1 and GmPGIP3 have been built using the crystal structure of PvPGIP2 as the template. The crystal structure of FmPG being available, the three PGIPs have been subsequently docked to FmPG. In order to elucidate the mechanism underlying and the strategy undertaken by the plants in utilizing the different isoforms of PGIP, a comparative study of the interaction between these three pairs namely PvPGIP1 and FmPG, PvPGIP2 and FmPG and GmPGIP3 and FmPG have been accomplished. A comparison of the binding modes, the interacting residues and the electrostatic potential surface along the interface of the PGIP-PG complex have been investigated. Change in intermolecular distances, hydrogen bond formation and electrostatic surface potential upon in silico mutation of PvPGIP2 have also been explored.

\section{Results and discussion Sequence analysis \\ Domain search}

Domain search using Pfam [23] discloses that the three PGIP molecules (two from P. vulgaris and one from G. max) studied here share identical domain architecture consisting of one LRR_NT2 (a domain found specifically at the $\mathrm{N}$ terminal ends of leucine-rich repeat proteins) at the N-terminal end and nine leucine-rich repeats (R1R9) (Figure 2). Out of the nine LRRs, four have been identified as plant-specific (PS) LRRs (R3-R5, R9) using EMBOSS [24] with the pattern [ILVF]xx[ILVF]xx[ILVF]x [ILVF]xx[NTSC]x[ILVF] [TS]GxIPxx[ILVF]Gx.

\section{Pairwise alignment}

Pairwise alignment carried out in order to identify similarities between the PGIP molecules at the sequence level, shows only eight varying residue positions between PvPGIP1 and PvPGIP2 (shown by the vertical bars in Figure 2A) and 34 varying positions between PvPGIP2 and GmPGIP3 (shown in Figure 2C). Four out of the eight variations between PvPGIP1 and PvPGIP2 fall in the repeat regions and the rest reside in the nonLRR (intervening region between two repeats) region of 


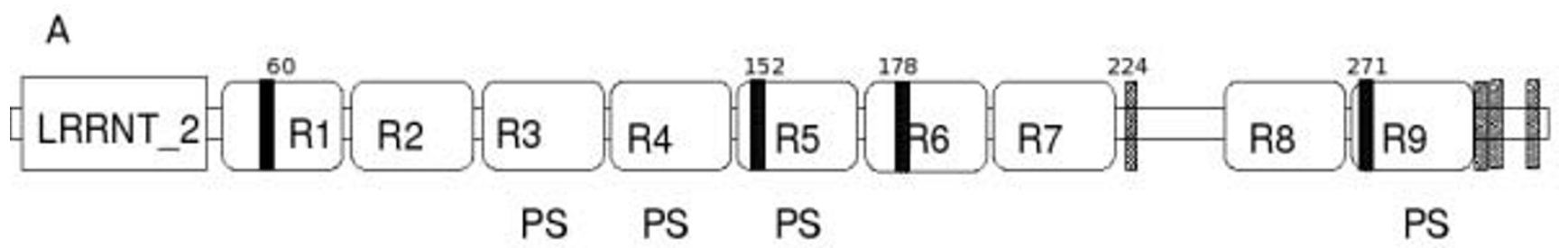

B
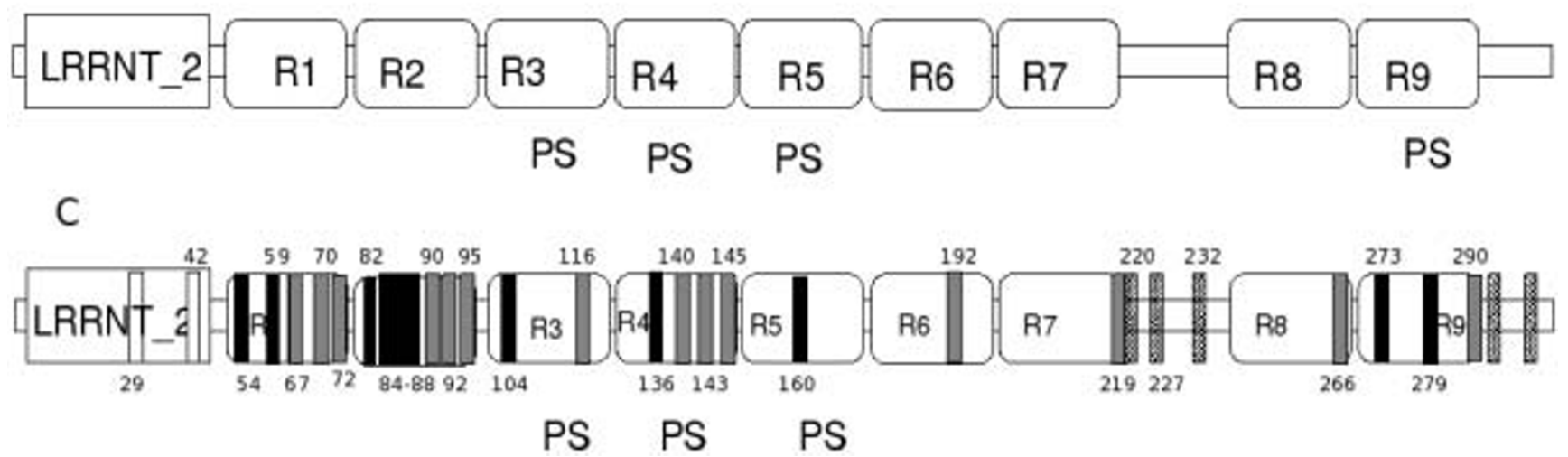

Figure 2

Domain architecture (DA) of the three PGIP molecules. A, B \& C show the DA of PvPGIPI, PvPGIP2 and GmPGIP3 respectively. Domains in all the three proteins are arranged as one LRR_NT2 domain followed by nine leucine-rich repeats (RI-R9). The domains are shown by rectangular boxes. Out of the nine LRRs, four have been marked as plant-specific (PS) LRRs (R3-R5, R9). The vertical bars in A and C denote the sequence variations with PvPGIP2 and the numbers stand for the residue number. The types of vertical bars change with the position of the varying residue, an information obtained from the multiple sequence alignments shown in Figure 3. Plain vertical bars stand for changes that are found in the LRR_NT2 region, the black and grey bars signify residue positions belonging to the conserved region and the variable region of LRRs respectively, while the hatched bars are for those in the non-LRR region.

the proteins. Two (R5 and R9) out of the four PS-LRRs and the first (R1) and the sixth (R6) repeat harbour these four variations mentioned above. The 34 variations between PvPGIP2 and GmPGIP3 on the other hand, are divided amongst all domains including LRRNT_2 and all the plant specific LRRs (Figure 2C).

\section{Distribution of the variations in the domains}

Out of the 34 residues that differ in GmPGIP3 from PvPGIP2 only two changes (Position 297 and 311 in Figure 1) are common with the 8 differing residues between PvPGIP1 and PvPGIP2 leaving the rest 32 variations to be at unique positions. 27 out of these 34 fall in the LRR regions and 2 in the LRRNT_2 region leaving altogether 5 positions to remain scattered in the intervening non-LRR regions. The sequence variation that fall in the LRR region in PvPGIP1(4) and GmPGIP3 (27) with PvPGIP2 could be distributed amongst the conserved portion of the repeat or the variable portion of it. It becomes an important task to determine this, as there are positions in the repeats where sequence variation is accepted without an associated change in structure. To account for the distribution of these variations with respect to different regions in the repeats, we constructed individual multiple sequence alignments. Figure 3 shows the multiple sequence alignment of nine repeats each from P $v$ PGIP1, PvPGIP2 and GmPGIP3. The conserved region of the repeats are marked by the conserved pattern of LRRs (written below each alignment) while the rest of the repeat signify its variable region. Considering an insertion of a residue $(G)$ in $R 2$ for PvPGIP1 and PvPGIP2 and an insertion of $\mathrm{P}$ in $\mathrm{R} 2$ of GmPGIP3 at another position, the variations (marked by black rectangles for those between PvPGIP2 and PvPGIP1 and grey rectangles for those between PvPGIP2 and GmPGIP3) can be rightly explained to be concentrated in positions (mostly X or variable region) where the motif itself has the provision for changes. The details of the distribution of the variations ( 8 for PvPGIP1 and 34 for GmPGIP3) in the LRR (conserved (c) and variable (v) region) and the non-LRR regions with reference to the residue type is tabulated in the Additional file 1. Depending on whether the variation is in the conserved 
A

R4

R8

R5

R2

R6

R9

R3

R1

R7

\section{B}

R4

R5

R8

R3

R9

R2

R6

R7

R1

C

R6

R9

R4

R5

R8

R7

R2

R3

R 1

\section{Figure 3}

Three individual multiple sequence alignments of the nine repeats of the PGIP molecules. $A, B$ and $C$ show the multiple sequence alignments with the nine LRR repeats (RI-R9) for PvPGIPI, PvPGIP2 and GmPGIP3 respectively.

The black coloured positions in $A$ and $B$ correspond to the residues that differ between PvPGIPI and PvPGIP2 while grey coloured positions in $B$ and $C$ highlight the varying residues between PvPGIP2 and GmPGIP3. The varying residue positions that are in bold are considered as insertion.

or variable part of the repeat or in the non-LRR region, black, grey and hatched vertical bars are respectively used in Figure $2 \mathrm{~A}$ and $2 \mathrm{C}$. In both these proteins (PvPGIP1 and GmPGIP3 that share a similarity of $87 \%$ ), most of the changes that fall in the LRR region (black bars in Figure 2) are either an allowed variant of the conserved position ( $\mathrm{L}$ or $\mathrm{C}$ or $\mathrm{N}$ ) or is any amino acid in the $\mathrm{X}$ position of the conserved region or is an amino acid belonging to the variable region of the repeat (grey bars). One exception is the variation in the conserved position $\mathrm{N}$ of R1 in PvPGIP1 (Figure 3A) where the replacing amino acid $\mathrm{H}$ is not an allowed variant. Regarding the variations in the non-LRR region, Additional file 1 points out that whereas PvPGIP1 and PvPGIP2 share 50\% of the changes in this region, GmPGIP3 and PvPGIP2 show only 15\% of it. This could probably indicate specific regions of importance in PGIPs for the introduction of variation for recognition of different enzymes from pathogens.

\section{Modelling}

The crystal structure of PvPGIP2 (1OGQ) being available and also since PvPGIP1 and PvPGIP2 as well as PvPGIP2 and GmPGIP3 share high percentage of sequence similarity, homology models of PvPGIP1 and GmPGIP3 were built using PvPGIP2 as template. One model each for PvPGIP1 and GmPGIP3 were selected using PROCHECK [25]. In each case, the model that was chosen supported the following criteria:

(i) more number of residues in the core region

(ii) no residues in the disallowed region

These models of PvPGIP1 and GmPGIP3 were solvated in water and were subjected to energy minimization followed by molecular dynamics simulation of 1 ns. A similar scheme of equilibration was taken up for the crystal structure of PvPGIP2. RMSD of the equilibrated structures of PvPGIP1 and GmPGIP3 were calculated with respect to the equilibrated structure of PvPGIP2 and was found to be $1.45 \AA$ and $1.66 \AA$ respectively.

\section{Docking}

We used GRAMM-X [26] for docking three pairs of proteins in order to unveil the mode of interaction within and across each pair. This docking program executes a rigid-body search using Fast Fourier Transform (FFT) correlation with simplified geometry employing shape complementarity and hydrophobicity in the scoring function. In this study we have used GRAMM-X as a protein-protein docking program as it extends the GRAMM Fast Fourier Transformation methodology by applying smoothed Lennard-Jones potential, refinement stage and knowledge-based scoring, thus giving rise to best surface match. The output of GRAMM-X is a PDB file containing the structures of ten models ranked as the most probable prediction candidate, according to the scoring function used. The cell-wall degrading enzyme PG, from the fungus Fusarium moniliforme was common 

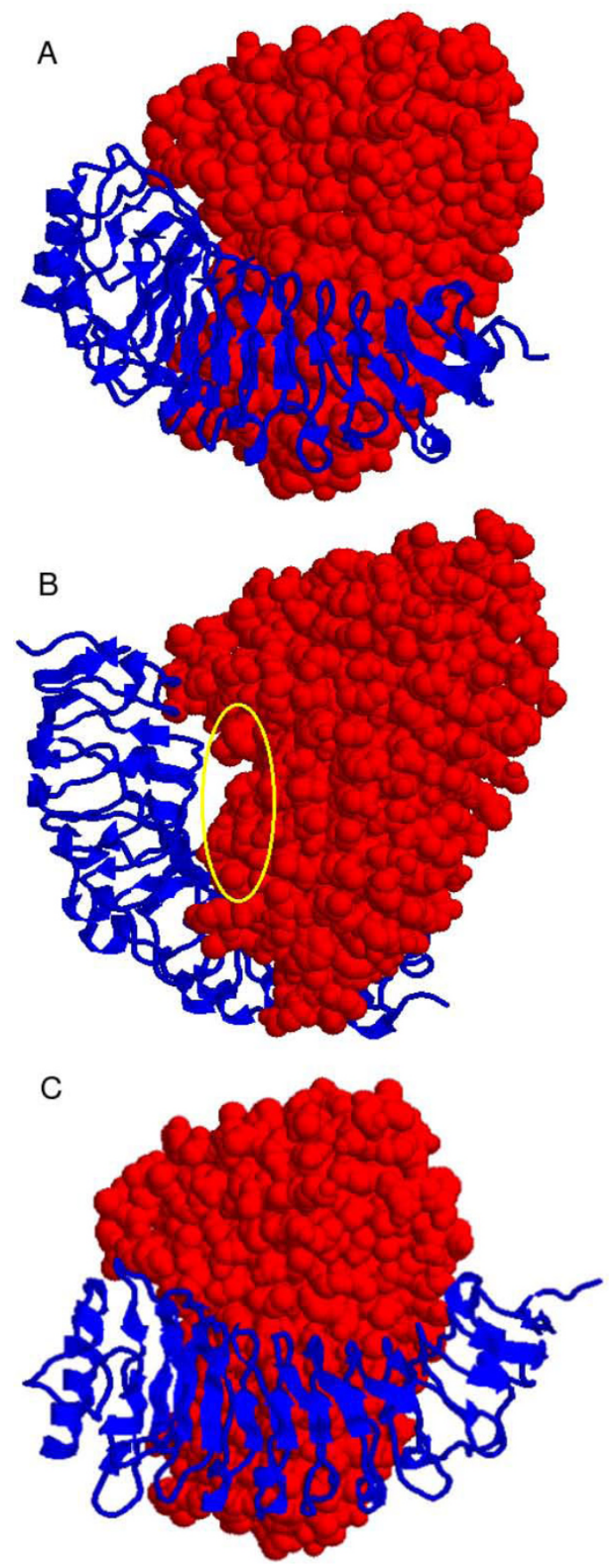

Figure 4

Docked complexes of PGIP (ribbon representation in blue) and FmPG (space-filling representation in red). A and $C$ show how PvPGIP2 (blue) and GmPGIP3 (blue) respectively, hinder the substrate binding site and block the active site cleft of FmPG (red). The only model of the PvPGIPI-FmPG complex where PvPGIPI (blue) docks near the active site of FmPG (red) although not blocking it unlike the other two complexes, is shown in B. The marked area shown by the yellow ellipse in $B$ indicates the portion of FmPG that PvPGIPI has been unable to block. in all the pairs and the other three molecules include $P v$ PGIP1, PvPGIP2 and GmPGIP3. The crystal structure of FmPG (1HG8) used in this study had also been solvated in water and was then subjected to energy minimization followed by molecular dynamics (MD) of $1 \mathrm{~ns}$.

\section{Docking studies of FmPG-PvPGIP2}

It has been reported that since P $v$ PGIP2 inhibits FmPG, hence one would expect PvPGIP2 to interact with PG by blocking the active site and/or the substrate binding cleft of the fungal enzyme. We obtained top scoring ten docked complexes of PvPGIP2-FmPG from GRAMM-X. One model in the exact expected orientation was selected on the basis of the existing experimental studies (see below). In the docked complex (Figure 4A), PvPGIP2 has covered the active site cleft by hindering the substrate binding site of FmPG. This is evident from the change in Solvent Accessible Surface Area (SASA) (shown in Table 1) for the residues Asp-167, Asp-188, Asp-189, Arg-243, and Lys-245 of FmPG that are known to be located inside the deep cleft and form the putative active site [27]. Functional studies confirm the involvement of these residues in the enzymatic reaction mechanism and or binding to the substrate which is in agreement with the reported mode of competitive inhibition with FmPG [27].

In this model structure, the residues of PvPGIP2 that is known to be functionally important, have shown considerable change in SASA (Table 1), indicating that they play a key role in interaction. Four residues [V (152), S(178), Q(224) and $\mathrm{H}(271)$ ] out of five that were identified by Leckie et al. [16] as interacting residues of $P v$ PGIP2 are expected to reside in the interacting surface of $P v$ PGIP2 in the PvPGIP2-FmPG complex, inferred on the basis of the considerable change in SASA (Table 1).

\section{Docking studies of FmPG-PvPGIPI}

Out of the ten potential candidates for models of $P v$ PGIP1-FmPG complex obtained from GRAMM-X, only in one model PvPGIP1 docked to the same face of FmPG as was found in the model for the complex of PvPGIP2-FmPG (Figure 4B). In the rest of the nine models, the PGIP molecule docked to a different face of FmPG indicating a gross inability of these models of $P v$ PGIP1 to block either the substrate binding site or the active site of FmPG. In the selected model, whereas the portion marked with the yellow ellipse is evidently seen to be blocked in the other two complexes, the same is not the case with PvPGIP1. This is in complete agreement with the experimental observation of PvPGIP1 being incapable of inhibiting the PG from Fusarium moniliforme. This model in which FmPG docks to PvPGIP1 at 
Table I: Change in Solvent Accessible Surface Area (SASA) in PGIP and PG upon complexation: The table summarizes the change in solvent accessible surface area of some biologically important residues of PvPGIP2-FmPG and GmPGIP3-FmPG before and after complexation

Change in SASA (in $\mathrm{nm}^{2}$ ) upon complexation of PvPGIP2 with FmPG

\begin{tabular}{llll}
\hline Residues in PvPGIP2 & & & \\
\hline Residue No. & Residue name & SASA in PvPGIP2 & SASA in complex \\
\hline 152 & $\mathrm{~S}$ & 0.726572 & 0.363286 \\
178 & $\mathrm{Q}$ & 0.264561 & 0.11834 \\
224 & $\mathrm{H}$ & 0.496097 & 0.137248 \\
$27 \mathrm{I}$ & & 1.01276 & 0.585858 \\
\hline Residues in FmPG & & & SASA in complex \\
\hline Residue No. & Residue name & SASA in PvPGIP2 & 0.306561 \\
\hline 167 & $\mathrm{D}$ & 0.348913 & 0.141431 \\
188 & $\mathrm{D}$ & 0.381939 & 0.0896238 \\
189 & $\mathrm{D}$ & 0.150885 & 0.0127235 \\
243 & $\mathrm{R}$ & 0.158454 & 0.510823 \\
245 & $\mathrm{~K}$ & 0.403263 & \\
\hline
\end{tabular}

Change in SASA (in $\mathrm{nm}^{2}$ ) upon complexation of GmPGIP3 with FmPG

\section{Residues in GmPGIP3}

\begin{tabular}{llll}
\hline Residue No. & Residue name & SASA in GmPGIP3 & SASA in complex \\
\hline 152 & V & 0.396312 & 0.066052 \\
178 & $\mathrm{~S}$ & 0.4249 & 0.182517 \\
224 & $\mathrm{Q}$ & 0.517371 & 0.29529 \\
225 & $\mathrm{~K}$ & 0.6919 & 0.1325 \\
$27 \mathrm{H}$ & $\mathrm{H}$ & 1.21099 & 0.712258 \\
\hline
\end{tabular}

Residues in FmPG

\begin{tabular}{llll}
\hline Residue No. & Residue name & SASA in GmPGIP3 & SASA in complex \\
\hline 167 & D & 0.348913 & 0.292443 \\
188 & D & 0.381939 & 0.207483 \\
189 & D & 0.150885 & 0.033026 \\
243 & R & 0.158454 & 0.0254469 \\
245 & K & 0.403263 & 0.523546 \\
\hline
\end{tabular}

the face similar to the one in PvPGIP2 (Figure 4B) of the Pv PGIP2-FmPG complex, is considered for further studies to confirm the inability of the protein in inhibition towards the enzyme.

\section{Docking studies of FmPG-GmPGIP3}

It has been reported that GmPGIP3 possesses an inhibiting activity towards the PG of Fusarium moniliforme like PvPGIP2. No mutational studies being available for GmPGIP3, the docked structure of the GmPGIP3-FmPG complex was therefore selected from the top scoring ten models reported by GRAMM-X using the knowledge obtained from experimental studies carried out for PvPGIP2. In the selected model of the complex (Figure 4C), GmPGIP3 has blocked the active site cleft of FmPG. The docked model therefore is in agreement with the experimental observation of the ability of GmPGIP3 in inhibiting the enzymatic activity of FmPG. The important residues V(152), S(178), Q (224) and H(271) of GmPGIP3 that are also present in PvPGIP2 show considerable decrease in solvent accessible surface area (Table 1) indicating their active involvement in protein-protein interaction, an identical observation that holds true for PvPGIP2. Thus like PvPGIP2, GmPGIP3 too blocks the active site cleft and hinders the substrate binding site of FmPG bearing a similar mode of competitive inhibition.

\section{Comparative study of protein-protein interactions in the three complexes \\ lonic interactions}

We have used PIC [28] for comparison of interactions recognized in the three complexes. Ionic interaction that is believed to play an important role in protein-protein 
interactions were studied for the three complexes namely PvPGIP2-FmPG, PvPGIP1-FmPG and GmPGIP3-FmPG. Table 2 shows that whereas this kind of interaction plays a vital role both in PvPGIP2-FmPG and GmPGIP3-FmPG complexes, it is not so for the PvPGIP1-FmPG complex.

\section{Electrostatic surface potential of individual PGIPs and the complexes}

Adaptive Poisson-Boltzmann Solver (APBS) package [29] was used to generate the electrostatic surface potential for each of PvPGIP1, PvPGIP2, GmPGIP3 and FmPG, as shown in Figure $5 \mathrm{~A}, \mathrm{~B}, \mathrm{C}$ and $5 \mathrm{D}$ respectively. It is evidently clear that the electrostatic surface potential for PvPGIP2 and GmPGIP3 (Figure 5B \&5C) show similarity along the surface at the concave face which is the site for interaction with FmPG. PvPGIP1 shows a distinctly different potential surface at a region in this face, even though it bears very few variations at the sequence level when compared to PvPGIP2.

Figure 6 shows the electrostatic potential surface and the mode of binding for the three complexes namely PvPGIP1-FmPG (Figure 6A), PvPGIP2-FmPG (Figure 6B) and GmPGIP3-FmPG (Figure 6C). The potential surface of the complex in PvPGIP1-FmPG is undoubtedly dissimilar from the other two complexes and so is the mode of binding. Interestingly, the mode of binding of GmPGIP3 with FmPG very much resembles that of $P v$ PGIP2 and FmPG although the sequence variation between the two PGIPs is significant.

\section{In silico mutation of PvPGIP2 and PvPGIP2-FmPG complex}

PvPGIP2 was mutated at six positions, with the amino acid present in PvPGIP1 at that position, using WHAT IF [30]. Electrostatic surface potential was generated for all the six mutated PvPGIP2 molecules. The surface drawn with the Q224K mutation showed considerable change from the wild type protein (Additional file 2). The other single mutations did not show much of a significant
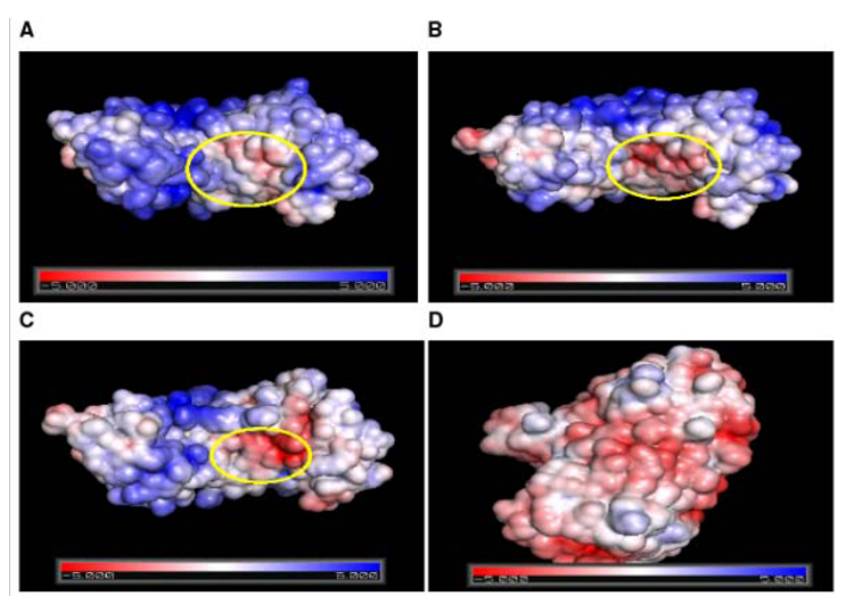

Figure 5

Electrostatic surface potential of the individual proteins. A, B, C \& D show the electrostatic surface potential on solvent accessible surface around PvPGIPI, PvPGIP2, GmPGIP3 and FmPG respectively at which the surface colors are clamped at red $(-5)$ or blue $(+5)$. The negatively charged region marked in $B$ and $C$ signify the area where they interact with their partner FmPG. The corresponding region in $A$ is distinctly different with less electronegativity in surface electrostatics in comparison to $B$ and $\mathrm{C}$.

change in the electrostatic surface potential. It may be mentioned that when these mutated PvPGIP2 molecules were docked to FmPG, none of the complexes showed any resemblance to the conformation found for the $P v$ PGIP1-FmPG complex, shown in Figure 6A.

When in the PvPGIP2-FmPG complex, Q (224) of PvPGIP2 was mutated to K, using WHAT IF, the resultant complex showed quite a few of the following deviations from the complex with the wild type protein. Hard sphere contact distances [31] between heavy atoms revealed an existence of steric clash between the $\mathrm{N} \zeta$ of Q224K of the mutated PvPGIP2 and C $\varepsilon$ of K(300) of FmPG. Whereas the contact distance between the atoms

Table 2: lonic interaction in PvPGIP2-FmPG, PvPGIPI-FmPG and GmPGIP3-FmPG complexes: The ionic interaction as obtained from Protein Interaction Calculator, showing the positions of the residue pairs in PvPGIP2-FmPG, PvPGIPI-FmPG and GmPGIP3-FmPG docked complexes

\begin{tabular}{|c|c|c|c|c|c|}
\hline \multicolumn{2}{|c|}{$\begin{array}{l}\text { lonic interaction showing the positions } \\
\text { of residue pairs in the PvPGIP2-FmPG } \\
\text { complex for }\end{array}$} & \multicolumn{2}{|c|}{$\begin{array}{l}\text { lonic interaction showing } \\
\text { the positions of residue pairs in the } \\
\text { PvPGIPI-FmPG complex for }\end{array}$} & \multicolumn{2}{|c|}{$\begin{array}{l}\text { lonic interaction showing } \\
\text { the positions of residue pairs in } \\
\text { the GmPGIP3-FmPG complex for }\end{array}$} \\
\hline PvPGIP2 & FmPG & PvPGIPI & FmPG & GmPGIP3 & FmPG \\
\hline $\begin{array}{l}46 \\
110 \\
203\end{array}$ & $\begin{array}{l}336 \\
146 \\
183\end{array}$ & 46 & 292 & $\begin{array}{c}56 \\
85 \\
131 \\
225 \\
294\end{array}$ & $\begin{array}{l}152 \\
146 \\
183 \\
180 \\
116\end{array}$ \\
\hline
\end{tabular}



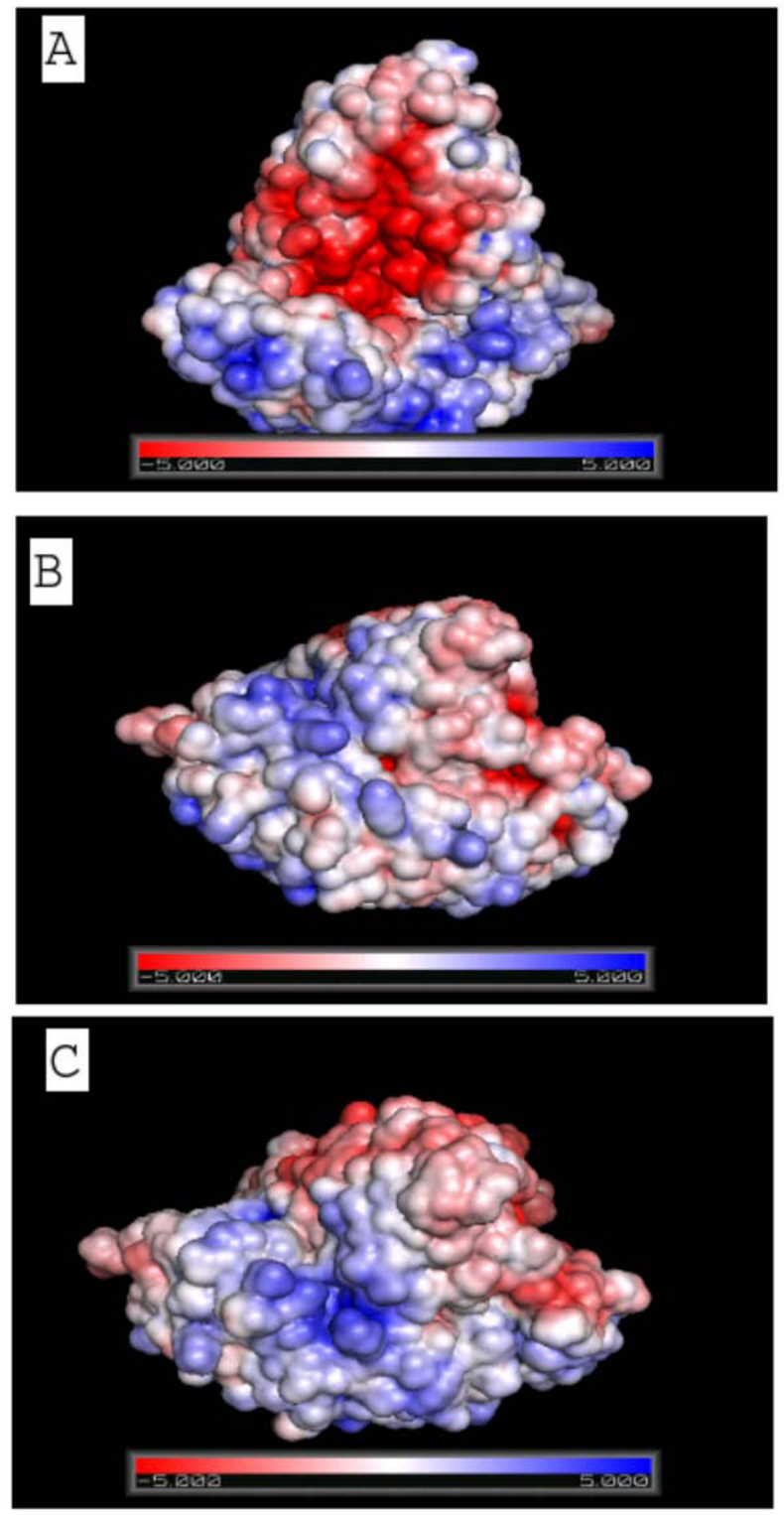

Figure 6

Electrostatic surface potential of the three complexes. A, B \& $C$ show the electrostatic surface potential on solvent accessible surface around PvPGIPIFmPG, PvPGIP2-FmPG, GmPGIP3-FmPG docked complexes respectively at which the surface colors are clamped at red $(-5)$ or blue (+5). B and C signify similar mode of binding and electrostatic interaction between the two PGIP molecules and FmPG whereas the mode of interaction between PvPGIPI and FmPG shown in A is strikingly different from the other two.

should be more than $2.52 \AA$, the distance in the complex with mutation was $2.47 \AA$. The complex bearing the mutation also showed the absence of the predicted hydrogen bond involving $\mathrm{Q}(224)$ of PvPGIP2 as obtained from PIC results. Even the ionic interaction that prevails in the wild type complex of PvPGIP2-FmPG vanishes with this single mutation. The in silico mutation study, confirms the importance of the $\mathrm{Q}(224)$ residue which has been experimentally found to give rise to a $70 \%$ reduction in the inhibition ability of the protein upon single mutation. The study also suggests probable reasons like a different electrostatic potential surface, steric clash or lack of hydrogen bonds that might explain why PvPGIP1 with only eight residues has a different mode of binding to FmPG.

The above in silico study clearly portrays how a single mutation in PvPGIP2 results in disallowed hard sphere contact distances between the two protein molecules thus indicating the importance of van der Waals interaction in plant defense. $Q$ at the $224^{\text {th }}$ position which is also involved in forming hydrogen bonds, plays a substantial role in this interaction. Since GRAMM-X shows that the mode of binding of PvPGIP1 with FmPG is different from PvPGIP2 and GmPGIP3 and further more since GRAMM-X has a major involvement of smoothed Lennard-Jones potential in scoring, we infer that the discrimination in recognition has a significant contribution from van der Waals interaction. Role of van der Waals forces have already been shown to be important in offering stability to several protein-ligand and in some cases DNA-ligand complexes prevailing in biological systems. Molecular Dynamics studies have shown van der Waals interaction to play a vital role in groove binding of the repressor fragment of a bacteriophage to its operator oligonucleotide [32]. In a study with proteins, it has been shown that the mouse major urinary protein (MUP) and its ligand show a binding thermodynamics that is dominated by dispersion interactions [33]. The involvement of the van der Waals forces in plant defense although not in specific recognition has also been reported. Many plants respond to pathogenic attack by producing defense proteins that bind to chitin, a polysaccharide present in the cell wall of the invading fungus or exoskeleton of infecting insects. NMR studies show the involvement of van der Waals interactions between the methyl acetamide group of the GlcNAc unit and a hydrophobic patch of the plant protein [34]. Studies on the characteristics of the sugar-binding sites in plant and animal lectin show van der Waals interaction and hydrogen bonds to contribute significantly. Selectivity for different sugars result from hydrogen bonds and unwanted recognition is prevented by steric exclusion [35]. Here in the case of plant defense through PGIP, the influence of van der Waals interaction therefore does not seem to be atypical.

In the present study, we have failed to address pertinent components like the change in the binding free energy 
change $(\Delta \Delta \mathrm{G})$ between the wild type and the mutated PGIPs. In order to obtain experimentally relevant information concerning the thermodynamic properties of binding, we wish to work in future, with advanced simulation methods such as free energy perturbation or thermodynamic integration to assess the binding free energy changes upon mutation of the important residues in PGIP.

\section{Conclusion}

It has been noted from our study on sequence analysis that $50 \%$ of the sequence variation between PvPGIP1 and PvPGIP2 fall in the non-LRR region whereas only $15 \%$ of the variation in GmPGIP3 with PvPGIP2 fall in the non-LRR region. This observation suggests specific regions in PGIPs favourable for the introduction of variation in recognition of the pathogen molecules. Structural studies involving docking techniques suggest the mode of binding of the fungal enzyme FmPG by PGIP2 from Phaseolus vulgaris to be similar to that of its homologue PGIP3 from Glycine max. In each case of binding, the active site of the enzyme from Fusarium moniliforme is being blocked by the PGIP molecule, supporting the experimentally observed phenomenon of these PGIP molecules being able to inhibit the fungal enzyme upon invasion. PGIP1 from the same plant Phaseolus vulgaris which is incapable of inhibiting FmPG, binds to FmPG in an evidently different mode, although $P v$ PGIP1 and PvPGIP2 share a higher degree of sequence similarity compared to PvPGIP2 and GmPGIP3. Electrostatic surface potential reveals considerable difference in the interacting surface of PvPGIP1 when compared to PvPGIP2 or GmPGIP3. Thus, electrostatic and van der Waals interactions may play a significant role in PGIPs for proper recognition and discrimination of PGs.

\section{Method}

\section{Sequence analysis}

The sequences of the three PGIPs, namely PvPGIP1, $P v$ PGIP2 and GmPGIP3 were retrieved from NCBI. We used the Pfam domain search program [23] to determine the domain architecture of these proteins. The plant specific Leucine-rich Repeats (PS_LRR) in the proteins were identified by the program PATMATDB of EMBOSS [24] using the pattern [ILVF] $\mathrm{xx}$ [ILVF] $\mathrm{xx}$ [ILVF] [ [LVF] $\mathrm{xx}$ [NTSC]x [ILVF] [TS]GxIPxx[ILVF]Gx as a regular expression. Pairwise alignments, one between PvPGIP1 and PvPGIP2 and another between GmPGIP3 and PvPGIP2 were carried out by the program NEEDLE of EMBOSS. All the nine repeats identified by Pfam were aligned by CLUSTALW [36] for the three proteins, individually.

\section{Modelling}

Homology models of PvPGIP1 and GmPGIP3 were generated using MODELLER 9v4 [37] by satisfying the spatial restraints, based on the homology they share with the protein PvPGIP2 (PDB Id: 1OGQ). The best model was selected using PROCHECK [25] based on steric correctness. Energy minimization was performed for all the structures that were involved in our studies, with the help of GROMACS 3.3.3 [38,39].

The four protein molecules namely, the models of PvPGIP1 and GmPGIP3 and the crystal structure of $P \nu$ PGIP2 and the fungal enzyme FmPG (PDB Id: 1HG8) were embedded in a box of simple point charge water molecules with $1 \mathrm{~nm}$ separation between the box boundary and the solute. Energy minimization of solvent with the solute fixed was carried out with 500 steps of steepest descent followed by 2000 steps of conjugate gradient algorithm. After that the constraint on the solute was released and energy minimization of the whole system was carried out again with 500 steps of steepest descent followed by 2000 steps of conjugate gradient algorithm. The resulting structures were subjected to 1 ns of molecular dynamics (MD) simulations in GROMACS at $300 \mathrm{~K}$. For all the simulations GROMOS96 43a1 force field was used.

The model structures of PvPGIP1 and GmPGIP3 have been deposited to PMDB Protein Model Database http:// mi.caspur.it/PMDB/ with the PMDB identifier of PM0075783 and PM0075784 respectively.

\section{Docking studies}

We have used GRAMM-X Protein Docking Web Server v.1.2.0 [26] for docking the three PGIP molecules to PG taken one at a time. The output PDB file that GRAMM-X produces contains 10 models ranked as the most probable prediction candidates according to the scoring function used by the program. The PGIPs acted as receptors while the PG acted as ligand in this study of protein-protein interaction. To select a model out of the ten top scoring docked complexes that GRAMM-X reported, we studied the associated change in Solvent Accessible Surface Area (SASA) of the amino acid residues in the individual molecules and when they are in the complex. We used the g_sas program in GROMACS to estimate the change in SASA for the individual residues of the proteins.

\section{Mutation prediction studies}

We have used mutation prediction tool [40] of the WHAT IF [30] web interface to mutate Q (224) of $P v$ PGIP2 to K in the PvPGIP2-FmPG complex. The same mutation $(\mathrm{Q} 224 \mathrm{~K})$ and five others that differ in $P v$ PGIP1 were carried out on PvPGIP2 by using the same server. 
Determination of ionic interaction and hydrogen bonding patterns in the complexes

The Protein Interaction Calculator (PIC) [28] was used to detect the ionic interaction between the proteins in the three complexes.

\section{Electrostatic surface potential}

Adaptive Poisson-Boltzmann Solver (APBS) is a software package for the numerical solution of the PoissonBoltzmann equation (PBE), one of the most popular continuum models for describing electrostatic interactions between molecular solutes in salty, aqueous media. The colour coded electrostatic surface potential was drawn using the APBS (Adaptive Poisson-Boltzmann Solver) package [29] within PYMOL 0.99rc6 [41] for the three PGIP molecules, the PG molecule and the three complexes. Electrostatic surface potential was also generated for a model where a single mutation was carried out on PvPGIP2 (Q224K) by WHAT IF $[30,40]$ using the APBS package [29].

\section{List of abbreviations used}

LRR: Leucine-rich repeat; PGIP: PolyGalacturonase Inhibiting Protein; pgip: PolyGalacturonase Inhibiting Protein coding gene; PG: PolyGalacturonase; PvPGIP: Phaseolus vulgaris PolyGalacturonase Inhibiting Protein; GmPGIP: Glycine max PolyGalacturonase Inhibiting Protein; FmPG: Fusarium moniliforme PolyGalacturonase; PAMP: pathogen associated molecular pattern; HGA: homogalacturonan; PS_LRR: plant specific Leucine-rich Repeats; SASA: Solvent Accessible Surface Area; APBS: Adaptive Poisson-Boltzmann Solver; PBE: Poisson-Boltzmann equation; PIC: Protein Interaction Calculator.

\section{Competing interests}

The authors declare that they have no competing interests.

\section{Authors' contributions}

Both AM and SB designed the plan of work and performed the analyses. HG participated in the development of the work. SB conceived of the study and coordinated the work. All authors read and approved the final manuscript.

\section{Note}

Other papers from the meeting have been published as part of BMC Bioinformatics Volume 10 Supplement 15, 2009: Eighth International Conference on Bioinformatics (InCoB2009): Bioinformatics, available online at http://www.biomedcentral.com/1471-2105/10? issue $=\mathrm{S} 15$.

\section{Additional material}

\section{Additional file 1}

Details of the distribution of the sequence variations in PvPGIP1 and GmPGIP3 with PvPGIP2 over the conserved and variable portion of the repeat and the non-LRR region. The table shows the distribution of the varying residues in PvPGIP1 and GmPGIP3 with PvPGIP2 in the LRRs and non-LRR regions. The changes that occur in the LRR region is either $L / N / C$ of the conserved segment ( $L x x L x L x x N x L$ ) or is $x$. They are denoted by $c L, c N, c C$ and $c X$ where $X$ is any amino acid. Amino acid in the variable region of the LRR is denoted by $v$.

Click here for file

[http://www.biomedcentral.com/content/supplementary/14712164-10-S3-S19-S1.pdf]

\section{Additional file 2}

Electrostatic surface potential of PvPGIP2 with a single mutation at 224. Q(224) of PvPGIP2 is mutated to K which is the residue found in PvPGIP1. The electrostatic surface potential changes considerably with a single mutation when compared to Figure 5B. Experimental studies on this single mutation have shown a $70 \%$ reduction in the inhibition ability of PvPGIP2.

Click here for file

[http://www.biomedcentral.com/content/supplementary/14712164-10-S3-S19-S2.pdf]

\section{Acknowledgements}

AM is grateful to Department of Biotechnology, Government of India for providing her with fellowship. The authors deeply acknowledge Dr. Ansuman Lahiri and Dr. Anindita Seal for their critical reading of the manuscript and useful discussion.

This article has been published as part of BMC Genomics Volume 10 Supplement 3, 2009: Eighth International Conference on Bioinformatics (InCoB2009): Computational Biology. The full contents of the supplement are available online at http://www.biomedcentral.com//47/-2/64/I0? issue $=\mathrm{S} 3$.

\section{References}

I. Federici L, Di Matteo A, Fernandez-Recio J, Tsernoglou D and Cervone F: Polygalacturonase inhibiting proteins: players in plant innate immunity? TRENDS in Plant Science 2006, I I:65-70.

2. Gomathi $\vee$ and Gnanamanickam SS: Polygalacturonase-inhibiting proteins in plant defense. Current Science 2004, 87: $1211-1217$.

3. De Vries RP and Visser J: Aspergillus enzymes involved in degradation of plant cell wall polysaccharides. Microbiol Mol Biol Rev 2001, 65:497-522.

4. Gao S and Shain L: Activity of polygalacturonase produced by Cryphonectria parasitica in chestnut bark and its inhibition by extracts from American and Chinese chestnut. Physiol Mol Plant Pathol 1995, 46:199-2।3.

5. Cervone F, Castoria R, Leckie F and De Lorenzo G: Perception of fungal elicitors and signal transduction. Signal Transduction in Plants Aducci P 1997, I53-177.

6. Robertsen B: Elicitors of the production of lignin-like compounds in cucumber hypocotyls. Physiol Mol Plant Pathol | 986, 28: | 37-|48.

7. D'Ovidioa R, Matteib B, Robertia S and Bellincampib D: Polygalacturonases, polygalacturonase-inhibiting proteins and pectic oligomers in plant-pathogen interactions. Biochimica et Biophysica Acta 2004, I696:237-244.

8. Bruce RJ and West CA: Elicitation of casbene synthetase activity in castor bean. Plant Physiol 1982, 69: | |8I-1 I88.

9. Cervone F, De Lorenzo G, Degra L and Salvi G: Elicitation of necrosis in Vigna unguiculata Walps Aspergillus niger 
endopolygalacturonase and a-D-galacturonate oligomers. Plant Physiol 1987, 85:626-630.

10. Kobe $B$ and Kajava $A V$ : The leucine-rich repeat as a protein recognition motif. Curr Opin Struct Biol 200I, II:725-732.

II. Kajava AV, Vassart G and Wodak SJ: Modeling of the threedimensional structure of proteins with the typical leucinerich repeats. Structure 1995, 3:867-877.

12. Ohyanagi T and Matsushima N: Classification of tandem leucinerich repeats within a great variety of proteins. FASEB J 1997, I I:A949.

13. Kajava AV: Structural diversity of leucine-rich repeat proteins. J Mol Biol 1998, 277:519-527.

14. Matteo AD, Federici L, Mattei B, Salvi G, Johnson KA, Savino C, De Lorenzo G, Tsernoglou D and Cervone F: The crystal structure of polygalacturonase-inhibiting protein (PGIP), a leucinerich repeat protein involved in plant defense. PNAS 2003, 100:10124-10128.

15. Roby D, Toppan A and Esurrre-Tugaye MT: Cell surfaces in plantmicroorganism interactions $\mathrm{V}$. Elicitors of fungal and of plant origin trigger the synthesis of ethylene and of cell wall hydroxyproline-rich glycoprotein in plants. Plant Physiol 1985, 77:700-704.

16. Leckie F, Mattei B, Capodicasa C, Hemmings A, Nuss L, Aracri B, De Lorenzo $G$ and Cervone $F$ : The specificity of polygalacturonaseinhibiting protein (PGIP): a single amino acid substitution in the solvent-exposed $b$-strand/b-turn region of the leucinerich repeats (LRRs) confers a new recognition capability. The EMBO Journal 1999, 18:2352-2363.

17. Ferrari S, Vairo D, Ausubel FM, Cervone F and De Lorenzo G: Tandemly duplicated Arabidopsis genes that encode polygalacturonase-inhibiting proteins are regulated coordinately by different signal transduction pathways in response to fungal infection. Plant Cell 2003, 15:93-106.

18. Powell AL, Kan J, Have A, Visser J, Greve LC, Bennett $A B$ and Labavitch JM: Transgenic expression of pear PGIP in tomato limits fungal colonization. Mol Plant Microbe Interact 2000, 13:942-950.

19. Aguero CB, Uratsu SL, Greve C, Powell ALT, Labavitch JM and Dandekar AM: Evaluation of tolerance to Pierce's disease and Botrytis in transgenic plants of Vitis vinifera L. expressing the pear PGIP gene. Mol Plant Pat 2005, 6:43-5I.

20. De Lorenzo $G$ and Ferrari S: Polygalacturonase-inhibiting proteins in defense against phytopathogenic fungi. Current Opinion in Plant Biology 2002, 5:295-299.

21. D'Ovidio R, Raiola A, Capodicasa C, Devoto A, Pontiggia D Roberti S, Galletti R, Conti E, O'Sullivan D and De Lorenzo G: Characterization of the complex locus of bean encoding polygalacturonase-inhibiting proteins reveals subfunctionalization for defense against fungi and insects. Plant Physiol 2004, I35:2424-2435.

22. D'Ovidio R, Roberti S, Di Giovanni M, Capodicasa C, Melaragni M, Sella L, Tosi $\mathrm{P}$ and Favaron F: The characterization of the soybean polygalacturonase-inhibiting proteins (Pgip) gene family reveals that a single member is responsible for the activity detected in soybean tissues. Planta 2006, 224:633-645.

23. Finn RD, Tate J, Mistry J, Coggill PC, Sammut SJ, Hotz HR, Ceric G, Forslund K, Eddy SR, Sonnhammer ELL and Bateman A: The Pfam protein families database. Nucleic Acids Research 2008, 36 : D28I-D288.

24. Rice P, Longden I and Bleasby A: The European Molecular Biology Open Software Suite. Trends in Genetics 2000, 16:276-277.

25. Laskowski RA, MacArthur MW, Moss DS and Thornton JM: PROCHECK: a program to check the stereochemical quality of protein structures. J Appl Cryst |993, 26:283-29|.

26. Tovchigrechko $A$ and Vakser IA: GRAMM-X public web server for protein-protein docking. Nucleic Acids Research 2006, 34: W3I0-4.

27. Federici L, Caprari C, Mattei B, Savino C, Matteo AD, De Lorenzo G, Cervone $F$ and Tsernoglou D: Structural requirements of endopolygalacturonase for the interaction with PGIP (polygalacturonaseinhibiting protein). PNAS 200I, 98:I3425-13430.

28. Tina KG, Bhadra $R$ and Srinivasan $N$ : Protein Interactions Calculator. Nucleic Acids Research 2007, 35:W473-W476.

29. Baker NA, Sept D, Joseph S, Holst MJ and McCammon JA: Electrostatics of nanosystems: application to microtubules and the ribosome. Proc Natl Acad Sci 200I, 98: 10037-1004|.

30. Vriend G: WHAT IF: a molecular modeling and drug design program. J Mol Graph 1990, 8:52-56.
31. Pappu RV, Srinivasan R and Rose GD: The Flory isolated-pair hypothesis is not valid for polypeptide chains: Implications for protein folding. PNAS 2000, 97: 12565-I2570.

32. Chang DK and Cheng SF: On the importance of van der Waals interaction in the groove binding of DNA with ligands: restrained molecular dynamics study. Int J Biol Macromol 1996, 19:279-285.

33. Barratt E, Bingham RJ, Warner DJ, Laughton CA, Phillips SE and Homans SW: van der Waals interactions dominate ligandprotein association in a protein binding site occluded from solvent water. J Am Chem Soc 2005, I 27: I | 827-II834.

34. Asensio JL, Canada FJ, Siebert HC, Laynez J, Poveda A, Nieto PM, Soedjanaamadja UM, Gabius HJ and Jimenez-Barbero J: Structural basis for chitin recognition by defense proteins: GICNAC residues are bound in a multivalent fashion by extended binding sites in hevein domains. Chem Biol 2000, 7:529-543.

35. Kulkarni KA, Sinha S, Katiyar S, Surolia A, Vijayan M and Suguna K: Structural basis for the specificity of basic winged bean lectin for the Tn-antigen: a crystallographic, thermodynamic and modelling study. FEBS Lett 2005, 579:6775-6780.

36. Thompson JD, Higgins DG and Gibson TJ: CLUSTAL W: improving the sensitivity of progressive multiple sequence alignments through sequence weighting, position specific gap penalties and weight matrix choice. Nucl Acids Res 1994, 22:4673-4680.

37. Sali $A$ and Blundell TL: Comparative protein modelling by satisfaction of spatial restraints. Mol Biol 1993, 234:779-815.

38. Berendsen HJC, Spoel van der D and van Drunen R: GROMACS: A message-passing parallel molecular dynamics implementation. Comp Phys Comm 1995, 91:43-56.

39. Lindahl E, Hess B and Spoel van der D: GROMACS 3.0: A package for molecular simulation and trajectory analysis. J Mol Mod 200I, 7:306-317.

40. Chinea G, Padron G, Hooft RWW, Sander C and Vriend G. The algorithm for the prediction of the conformation of the new side chain is described in: The use of position specific rotamers in model building by homology. Proteins 1995, 23:4|5-42|.

41. DeLano WL: The PyMOL Molecular Graphics System on World Wide Web. 2002 http://www.pymol.org.

Publish with BioMed Central and every scientist can read your work free of charge

"BioMed Central will be the most significant development for disseminating the results of biomedical research in our lifetime. "

Sir Paul Nurse, Cancer Research UK

Your research papers will be:

- available free of charge to the entire biomedical community

- peer reviewed and published immediately upon acceptance

- cited in PubMed and archived on PubMed Central

- yours - you keep the copyright
BioMedcentral 